\title{
HUMAN FACTOR IN DECISION-MAKING IN SIMULATION MODEL OF TRANSPORTATION SISTEM AND APPROACHES TO ITS MODELLING
}

The article deals with human factor in decision-making in simulation model of transportation system. It points to the difference between the management in reality and in a model, where it is more difficult to represent the human thinking. The consequence of this is a higher probability of deadlock appearances that can be developed after operation has deflected from the plan. The effort to minimize the operation deviations leads to processing larger amount of information and to more complicated decision-making algorithms, which makes the simulation model more complicated and slower. The task of the developer is to set the level of details in the decision-making subsystem.

In the end of the article, few different approaches to the modelling of human decision-making are mentioned.

\section{Introduction}

Computer simulation model of transportation system represents one of tools that help us in solving problems and making improvements in transportation systems. This tool may have a various level of capture of details from real transportation system - its master, depending mainly on what is it designed for.

When we focus on detailed analysis and improvement of technological processes, we may happen to work with complex simulation models containing hundreds of objects and thousands of parameters. In such models, management of interaction of objects gains high importance and more sophisticated decision-making is required.

In this paper, I'll discuss more in detail what problems in decision-making may be encountered when modelling a transportation system.

\section{Decision-Making in Simulation Model}

Simulation models intend to model real systems as closely as possible. Objects with their attributes in reality serve as masters for creation of objects in a simulation model. Relations among objects in a model also reproduce relations among objects in the master system. In this way also decision-making processes in the models are made based on decision-making processes in real systems.

There is one significant difference in decision-making in a real transportation system and in its simulation model: human factor. While it is people who make decisions in a real system, in its simulation model built on the computer, they are supposed to be made by the computer.
As we know, modelling of human thinking is not as an easy task as modelling of non-human objects and their behaviour. With growing complexity of the transportation system grows importance of human decision-making, and also difficulties in modelling it in a simulation model rise.

\section{Deadlock}

A major problem is appearance of deadlocks. Deadlock is a name for a situation when a process in the system is waiting for another process to be finished and at the same time, the latter process is waiting for the former process to be finished. Neither of them can finish, because they wait for each other. This happens commonly when more processes can use the same resources.

For instance (fig. 1), the locomotive A is moving from a locomotive depot to a train in the station, where it is assigned. At the same time, the locomotive B is moving from the station to the locomotive depot. Both locomotives are supposed to use the same route between the depot and the station, the former in one direction, the latter in the opposite direction. They get their routes assigned gradually, section by section, as they move. They are moving simultaneously until they come to a point, when next section in the route of the locomotive $\mathrm{A}$ is occupied by the locomotive $\mathrm{B}$ and

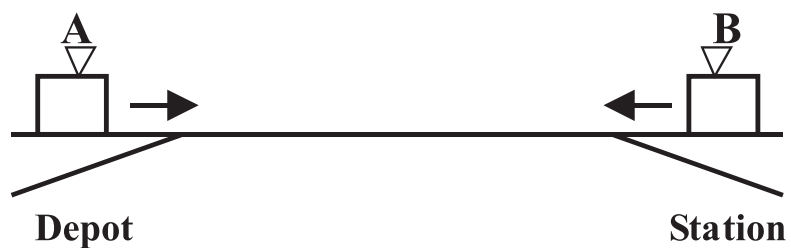

Fig. 1 Deadlock

\footnotetext{
* Ing. Michal Žarnay

Department of Transportation Networks, Faculty of Management Science and Informatics, University of Žilina, Moyzesova 20, 01026 Žilina, Slovak Republic, Tel: +421-41-5134224, E-mail: Michal.Zarnay@fri.utc.sk
} 
next section in the route of the locomotive B is taken by the locomotive A. They stop to wait for their resources to be assigned. And if they have no alternatives for movement, they can wait forever they are in deadlock.

\section{Plan and Management}

In most transportation systems (e.g. railway station, airport, local bus network), an operation is planned in advance. A plan assumes time points when events will happen and contains actions that should follow the events in context of current situation. Because of stochastic influence, events do not happen always according to the plan. Thus deviations from the plan appear - e.g. deviations in time or in selection of used resources.

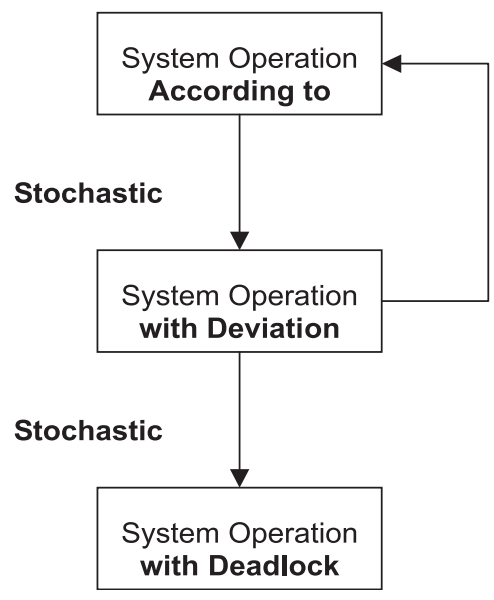

Operative Decisions

Fig. 2 Stochastic influence and operative management in transportation

In this situation, it is a task of an operative management subsystem to adapt the plan to the reality. It must take such actions that the system comes back to the plan. If there are enough resources to cope with the deviation (time reserves, available personnel or equipment), the management subsystem can remove the deviations and bring the system back to the plan. If any of required resources fails, the deviation may grow, and come to a deadlock eventually (fig. 2).

For instance, in a railway station, the plan is represented by a timetable that predicts events of departures and arrivals of trains. Based on this, plans of corresponding technological processes and services to passengers are developed. In this way, employees at the station know when the train should be shunted, cleaned, or inspected. Each employee has an individual working plan with a list of time points and train numbers to work on.

Stochastic influence causes that trains may run late, cargo trains may contain more loaded cars, or any cars for special manipulation, or unexpected failures may cause delay of technological operations.
The deviations in this example could be: using a track that was not planned for the given train, delay in processing of the train or using another personnel group for certain operation than originally planned.

\section{Problem in Modelling of Human Decision-Making}

In a real transportation system, the management subsystem contains human factor, which is able to spot deviations that may lead later to a deadlock. This person monitors the system and does necessary operations to keep it running closely to the plan.

In a simulation model, in most cases, the human being in the management subsystem is replaced by computer logic. The subsystem contains also tools for monitoring the system and for performing necessary operations. These are prescribed in a form of algorithms by a human being in advance. Each algorithm is supposed to make a decision in a given situation. The situation is recognised from available information about the current situation in the system. Based on it, a decision is made.

Quality of the decision depends on two factors: quality of processed information and quality of decision-making algorithm. The processed information must contain basic data that is absolutely necessary for the decision-making. It can also contain additional information that can influence quality of the solution. Similarly, the decision-making algorithm must contain some absolutely necessary minimum to make the requested decision. To ensure higher quality of solution, the algorithm may be also more sophisticated and contain mechanisms for processing additional information and evaluating additional criteria when choosing a decision.

Let's see an example (fig.3). Passenger train No. 340 comes to a train station delayed. According to the plan, it is supposed to use the platform track No. 3 in the station. However, at its delayed arrival another train No. 2801 should occupy the track. To solve this conflict, an additional track must be used.

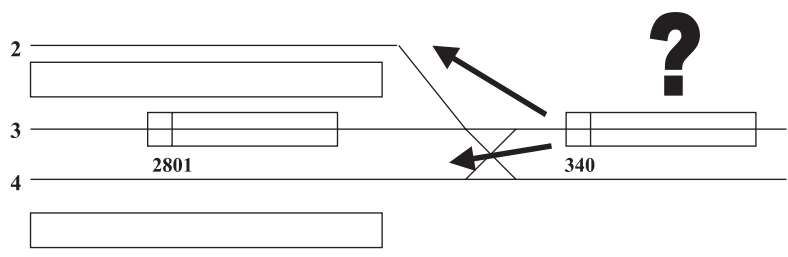

Fig. 3 Example of conflict situation

We have two options: platform tracks No. 2 or No. 4 . This information (list of additional tracks for use) is the necessary minimum to solve the situation. A simple algorithm may use the First-In-First-Out method. According to this the train No. 340, as coming later, will use the track No. 2, which is selected as the first in the list.

However, as consequence of this decision, the evident deviation from the plan may be spread further to other trains and tracks, 
because use of the track No. 2 will influence the processing of another train planned for that track. Another decision, use of the track No. 4 in this case, could have other consequences. It could spread the deviations from the plan to other parts of the model as well, more or less than the former decision. Our simple algorithm does not compare them.

If additional information about consequences was available or the decision-making algorithm processed available information in more sophisticated way, another solution could be chosen bringing the deviation closer to its end in near future. In this example, it could use information from the occupation plan of the platform tracks No. 2 and No. 4 in the next 10 minutes, or check how modified routes of trains through the station will influence other movements. This information could be compared with additional criteria for use of the platform tracks and for movements in the station planned in the near future. Results of this evaluation could contribute to the selection of one of the two alternatives.

It is obvious that making decisions of better quality would require more information in the phase of monitoring or more detailed processing of available information in the phase of decisionmaking (fig. 4). However, the more parameters a simulation model has, the larger is variety of different situations to solve, the more sensitive is model to stochastic events and probability of deadlocks is higher.

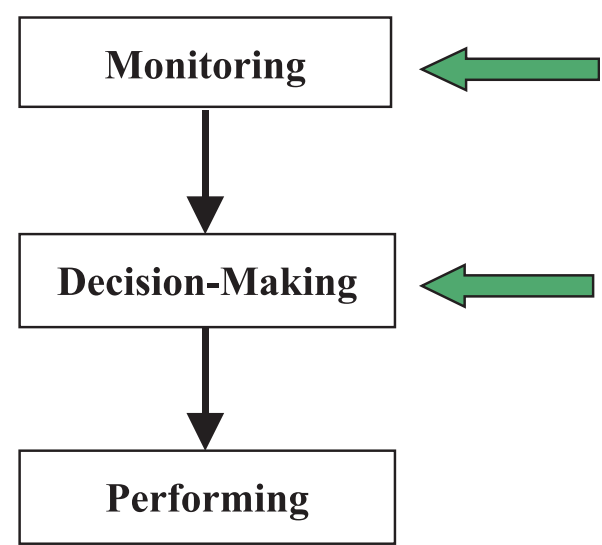

Fig. 4 Phases of focus when improving decision-making

In order to prevent a complex simulation model from deadlocks and to keep it in line with the plan, requirements on the computer management subsystem are higher which means a larger scale of information needed for decision-making and more complicated algorithms. Both aspects require deeper knowledge of management subsystem in the transportation system by a simulation model designer. Information from more sources in the model and more ways of combining it must be taken into account.

Apart from that decision-making processes last longer and require more computing power. This makes simulation models slower.

\section{Possible Approaches in Modelling of Human Decision-Making}

There is a wide range of possible approaches for solving this issue: from application of exact algorithms (e.g. from mathematical programming), through heuristic algorithms to application of artificial intelligence with fuzzy logic. It can be also a combination of all.

Exact algorithms can be used in only a small extent of situations, since their application usually requires unacceptable simplification of the problem or they need too long time to come to a solution, or both. That is why heuristic algorithms and artificial intelligence are applied in most of the cases.

In the described area, in our department, so far we have used the exact algorithms for solving simple problems. For complicated decision-making situations, heuristic algorithms are used. The development goes on and we are improving heuristic algorithms and investigating the area of artificial intelligence.

Another way that can help in solving, is modelling with help of Petri Nets. This formal tool allows not only modelling, but also simulation and analysis of the modelled system. There are different classes of Petri Nets used in different cases, e.g. coloured Petri Nets, timed Petri Nets or fuzzy Petri Nets.

An idea that we would like to investigate is to use coloured Petri Nets and their analysis space for solving individual decisionmaking situations that will be extracted from the modelled system. It is also possible that for representation of facts and rules for decision-making, fuzzy logic will be used.

\section{Conclusion}

A result of the development of management subsystem to a more complex version is its complexity and slower speed of the simulation model. This can harm some of the advantages of simulation models: speed and clarity.

In this way, a simulation model designer is between two extreme approaches leading to:

- On one side a faster and clearer model with simpler management subsystem, but with more difficult task of managing stochastic events in the model (deviations from plan),

- On the other side a more robust model able to deal with more various situations as results of stochastic events, but slower with more complex management subsystem.

It is a task for a designer to find a compromise between the two approaches and to define how close the compromise will be to any of them. This compromise depends most often on goal(s) carried out by simulation.

Possible approaches in modelling of human decision-making are various. One of them, connected with application of Petri Nets, is to be investigated. 


\section{References:}

[1] ŽARNAY, M.: Decision-making support in simulation models of nodes of railway network. Perner's Contact 2001, Pardubice, 2001

[2] ŽARNAY, M.: Analysis of decision-making in simulation model of node of railway network. Perner's Contact 2001, Pardubice, 2001

[3] KAVIČKA, A., KLIMA, V.: Interactive problem solving in simulation model of marshalling yard operation. In: Proceedings of the 3rd international symposium “ŽEL 96”, Žilina, 1996, pp. 229-233

[4] KLIMA, V., KAVIČKA, A.: Agent-based simulation model design. In: Proceedings of European simulation multiconference, SCS Budapest, 1996, pp. 254-258. 